\title{
ARTICLE
}

\section{Competing antiferromagnetic phases of the under-screened Anderson lattice model (INVITED)}

\author{
Ana C. Lausmann ${ }^{\mathrm{a}}$, Eleonir J. Calegari ${ }^{\mathrm{a}}$, Sergio G. Magalhaes ${ }^{\mathrm{b}}$ and Peter S. Riseborough ${ }^{\mathrm{c}^{*}}$ \\ ${ }^{a}$ Departamento de Fisica, Universidade Federal de Santa Maria, 97105-900, Santa Maria, RS, Brazil, ${ }^{b}$ Instituto de Fisica, \\ Universidade Federal do Rio Grande do Sul, 91501-970, Porto Alegre, RS, Brazil; ${ }^{c}$ Department of Physics, Temple University, \\ Philadelphia, PA 19122, USA
}

We investigate the magnetic phases of the Under-screened Anderson Lattice Model (UALM) for nearly half-filled $5 \mathrm{f}$ bands and find a competition between two distinct magnetic phases. The version of the UALM that we consider consists of two correlated $5 \mathrm{f}$ bands that, in the paramagnetic state, hybridize with a single conduction band. At the Néel temperature, the paramagnetic state undergoes a second-order transition to antiferromagnetically ordered states, in which the Néel order parameter induces a mixing between the states with different $5 \mathrm{f}$ characters. As pressure is applied, there is a line of first-order transitions that separates two Néel phases. Furthermore, as the pressure is increased, the Néel temperature decreases as if the system is being driven towards a quantum critical point. However, the nature of the paramagnetic instability changes from second-order to first-order before the Neel temperature reaches absolute zero.

Keywords: unders-creened Anderson lattice model; spin density waves; competing phases: phase diagram; uranium compounds;

\section{Introduction}

The Under-screened Anderson Lattice Model (UALM) has been used to describe the anomalous properties of several uranium compounds. The UALM is the natural generalization of the Under-screened Kondo Impurity Model (UKIM) for crystalline compounds. The UKIM describes localized moments $\mathrm{S}$ which interact with conduction electrons via an on-site antiferromagnetic interaction which, through the Kondo effect, induces a compensating antiparallel spin polarization of the conduction electrons that only partially quenches the moment on the impurity site [1]. This leads to a residual moment and hence, an entropy associated with the impurity at $\mathrm{T}=0$. The uranium monochalcogenides exhibit resistivities that follow logarithmic temperature dependencies at high temperatures, which indicates that Kondo scattering is at work. The logarithmic temperature dependence of the resistivities is interrupted at temperatures of the order of $100 \mathrm{~K}$, where the materials order magnetically [2,3]. For the ferromagnetic uranium monochalcogenides, the saturated ordered magnetic moments are consistent with partial Kondo compensation of the free uranium moments inferred from the high-temperature Curie-Weiss form of the susceptibilities. While some cerium compounds do exhibit signatures of the Kondo

*Corresponding author. Email: prisebor@temple.edu effect at high temperatures and undergo magnetic ordering at low-temperatures, the transition temperatures and the saturated ordered moments of the uranium compounds are orders of magnitude smaller than those of the corresponding cerium compounds [4]. Therefore, one may expect that the UALM may be appropriate to describe the uranium monochalcogenides [5,6]. Furthermore, one may expect that the UALM may also describe the antiferromagnetic ordering observed in the uranium-pnictides [7,8] and $\mathrm{UIrSi}_{3}$ [9]. It has been proposed that the UALM may describe the enigmatic "Hidden Ordered" phase of the itinerant $5 \mathrm{f}$ material $\mathrm{URu}_{2} \mathrm{Si}_{2}[10,11]$.

In this paper, we survey the antiferromagnetically ordered phases of the UALM for nearly half-filled $5 \mathrm{f}$ bands, as found by mean-field theory. We incorporate a direct $5 \mathrm{f}$ to $5 \mathrm{f}$ hopping matrix element in the UALM that introduces a delocalization of the residual $5 \mathrm{f}$ local moments. We investigate the pressure-temperature phase diagram by assuming that the main effect of applying pressure is to reduce the inter-atomic spacings and, thereby, increase the band-width which reduces the tendency for magnetic ordering.

\section{The Under-screened Anderson lattice model}

The under-screened Anderson Lattice Model, used in the present work, is described by the Hamiltonian 


$$
H=H_{f}+H_{d}+H_{f d}
$$

Where

$$
\begin{aligned}
& H_{f}=\sum_{\underline{k}, \sigma, \chi} E_{f}^{\chi}(k) f_{\underline{k}, \sigma}^{\dagger} f_{\underline{k}, \sigma} \\
& +\left(\frac{U-J}{2 N}\right)_{\underline{k},-k^{\prime}, \underline{q}, \sigma, \chi \neq \chi^{\prime}} f_{\underline{k}+\underline{q}, \sigma}^{\dot{\gamma}, \chi} f_{\underline{k}, \sigma}^{\chi} f_{\underline{k^{\prime}}-\underline{q}, \sigma}^{\dot{\gamma}, \chi^{\prime}} f_{\underline{k}^{\prime}, \sigma}^{\chi^{\prime}}
\end{aligned}
$$

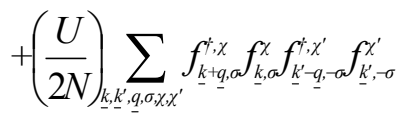

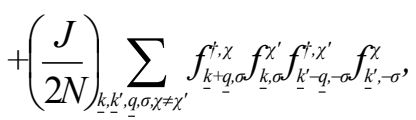

$$
\begin{aligned}
& H_{d}=\sum_{\underline{k}, \sigma} \varepsilon(\underline{k}) d_{\underline{k}, \sigma}^{\dagger} d_{\underline{k}, \sigma} .
\end{aligned}
$$

The dispersion relation of the conduction electrons is given by:

$$
\varepsilon(k)=-2 t\left[\cos \left(a k_{x}\right)+\cos \left(a k_{y}\right)+\cos \left(a k_{z}\right)\right]
$$

where $a$ is the lattice parameter, $\mathrm{W}=6 \mathrm{t}$ and $2 \mathrm{~W}$ is the total band-width. The hybridization tem is:

$$
H_{f d}=\sum_{\underline{k}, \sigma, \chi}\left(V_{\chi}(\underline{k}) f_{\underline{k}, \sigma}^{\dagger, \chi} d_{\underline{k}, \sigma}+V_{\chi}(\underline{k}) d_{\underline{k}, \sigma}^{\dagger} f_{\underline{k}, \sigma}\right)
$$

The first term in the Hamiltonian describes two $5 \mathrm{f}$ bands (labeled by $\chi=(\alpha, \beta)$ ), while the other terms represent the screened Coulomb interaction, $U$, and the Hund's rule interaction, $J$. The term $H_{d}$ describes the conduction band in which $\varepsilon(k)$ is the nearest-neighbor tight binding hopping energy and the $H_{f d}$ term represents the hybridization. We shall assume that $V_{\beta}(k)=0$, which does not affect the results. The first term $H_{f}$ is treated in the mean-field approximation, in which the fluctuations $\Delta \hat{n}_{f q \sigma}^{\chi}=\left(\hat{n}_{f q \sigma \sigma}^{x}-\left\langle\hat{n}_{f q \sigma .}^{x}\right)\right)$ are considered as being small when compared with the average value of $\hat{n}_{f q \sigma}^{\chi}$. The expectation value of $\hat{n}_{f \underline{q \sigma}}^{\chi}$ is re-written in terms of the Néel order parameters via

$$
\left\langle\hat{n}_{f q, \sigma}^{\chi}\right\rangle=\frac{n_{f}^{\chi}}{2} \delta_{\underline{q 0}}+\eta(\sigma) m_{f}^{\chi} \delta_{\underline{q, \pm \underline{Q}}}
$$

where $\mathrm{Q}=(\pi, \pi, \pi)$ and $\eta(\sigma)= \pm 1$ for $\sigma=\uparrow, \downarrow$ respectively. The quantity $\mathrm{m}_{f}^{x}$ is identified with the spin density wave (SDW) order parameter and satisfies a pair of coupled equations

$$
\begin{aligned}
& m_{f}^{\alpha}=\left(U m_{f}^{\alpha}+J m_{f}^{\beta}\right) \chi_{f}^{\alpha, \alpha}\left(\Delta_{\alpha}\right) \\
& m_{f}^{\beta}=\left(U m_{f}^{\beta}+J m_{f}^{\alpha}\right) \chi_{f}^{\beta, \beta}\left(\Delta_{\beta}\right)
\end{aligned}
$$

which involves the energy gaps $\Delta_{\alpha(\beta)}$

$$
\Delta_{\alpha(\beta)}=U m_{f}^{\alpha(\beta)}+J m_{f}^{\beta(\alpha)}
$$

The $\alpha$ susceptibility that appears in the above equations is given by

$$
\chi_{f}^{\alpha, \alpha}\left(\Delta_{\alpha}\right)=\frac{1}{4 \pi i N} \sum_{\underline{k}} \oint f(\omega) F^{\alpha \alpha}(\underline{k}+\underline{Q}) d \omega
$$

where $f(\omega)$ is the Fermi-function and the contour encircles the real axis. The function $F^{\alpha \alpha}(\underline{k}+Q)$ is related to the $5 \mathrm{f}$ Green's function $G^{\alpha \alpha}(\underline{k}+Q)$. The $\beta$ susceptibility is given by

$$
\chi_{f}^{\beta, \beta}\left(\Delta_{\beta}\right)=\frac{1}{2 N} \sum_{\underline{k}}\left[\frac{f\left(E_{\beta}^{+}(\underline{k})\right)-f\left(E_{\beta}^{-}(\underline{k})\right)}{\sqrt{(t \varepsilon(\underline{k}))^{2}}+\left(\Delta_{\beta}\right)^{2}}\right]
$$

in which

$$
E_{\beta}^{ \pm}(\underline{k})=\varepsilon_{f} \pm \sqrt{t^{2} \varepsilon^{2}(\underline{k})+\left|\Delta_{\beta}\right|^{2}} .
$$

The Helmholtz Free-Energy $F_{N}$ can be expressed in terms of the Néel order parameters as

$$
\begin{aligned}
& F_{N}=-k_{B} T \sum_{\gamma, \underline{k}} \ln \left(1+\exp \left[-\frac{\left(E^{\gamma}(\underline{k})-\mu\right)}{k_{B} T}\right]\right)+\mu N_{t} \\
& +\frac{N}{U^{2}+J^{2}}\left[U\left(\Delta_{\alpha}^{2}+\Delta_{\beta}^{2}\right)-2 J \Delta_{\alpha} \Delta_{\beta}\right]
\end{aligned}
$$

\section{Numerical results}

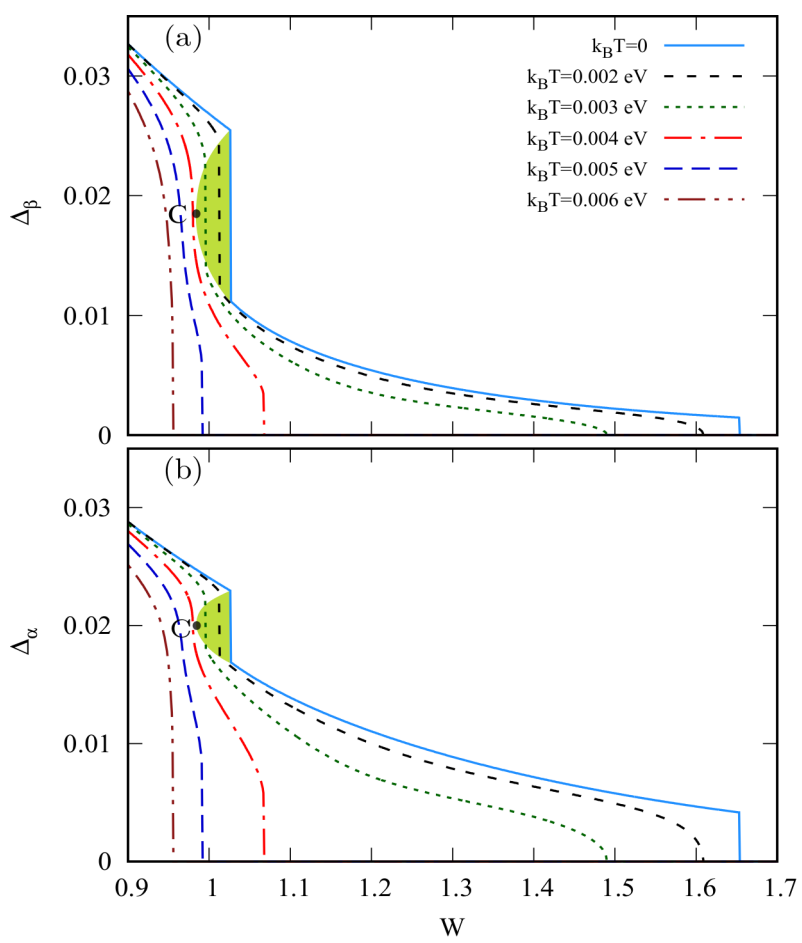

Figure 1. The upper panel (a) shows $\Delta_{\beta}$ as a function of the band-width $W$, for various temperatures. The lower panel (b) shows $\Delta_{\alpha}$ for the same range of temperatures and band-widths. 
The results presented in this section were calculated with the parameter values $U=5, J=0.165, V_{\alpha}=1 / 10$ and the value of the effective $5 \mathrm{f}$ level energy was taken to be $E_{f}=0.3 \mathrm{eV}$. The occupation number of the $5 \mathrm{f}$ bands was chosen as being close to 2 .

The gaps $\Delta_{\alpha}$ and $\Delta_{\beta}$ as a function of the conduction band-width (half-width) W, are shown in Figure 1 for different temperatures. Note that the increase of $W$ acts in the sense of suppressing the Néel state. Besides that, at low temperatures, for instance $k_{B} T=0, \Delta_{\beta}$ exhibits a discontinuity at $W \approx 1.03$. Indeed, near $W=1.03$, the system of equations has several solutions. The area highlighted by the green color shows the region where there are multiple solutions for $\Delta_{\alpha}$ and $\Delta_{\beta}$. The point C indicates the smallest value of $\mathrm{W}$ and the highest temperature, for which $\Delta_{\alpha}$ and $\Delta_{\beta}$ show discontinuous behavior. In the regions where the equations possess multiple solutions (shown in green), the Helmholtz Free-Energy has been used to determine which of the solutions for $\Delta_{\alpha}$ and $\Delta_{\beta}$ are more stable. The behavior of the gap $\Delta_{\alpha}$ is similar to that of $\Delta_{\beta}$, as can be seen in Figure 1(b).

The discontinuity exhibited by $\Delta_{\alpha}$ and $\Delta_{\beta}$ indicates that a first-order transition occurs between two competing SDW's, here called SDW1 and SDW2. The temperature at the point $\mathrm{C}$ is $k_{B} T \approx 0.004 \mathrm{eV}$. For $k_{B} T>$ $0.004 \mathrm{eV}$, the gaps $\Delta_{\beta}$ and $\Delta_{\alpha}$ vary continuously and go to zero at $T_{N}$. However, another interesting result is found at very low temperatures and large values of the band-width $W$. For $k_{B} T>0.001 \mathrm{eV}$, the phase transition between the SDW2 and the paramagnetic phase is a second-order type of phase transition. However, at $k_{B} T$ $=0$, both $\Delta_{\alpha}$ and $\Delta_{\beta}$ exhibit a discontinuous behavior indicating another first-order phase transition between the SDW2 and the paramagnetic phase. The replacement of a second-order transition between the SDW2 and the paramagnetic phase at higher temperature by a first-order transition at lower temperatures suggests the presence of a tri-critical point. This tri-critical point has been located at $k_{B} T \approx 0.001 \mathrm{eV}$.

The nature of the phase transition is illustrated by a complementary analysis of the behavior of $\Delta_{\alpha}$ and $\Delta_{\beta}$ as a function of temperature $T$. Figure 2 shows the gaps as a function of $T$, for several values of the band-widths $W$. For $W=0.90 \mathrm{eV}$ and $W=0.95 \mathrm{eV}$, both $\Delta_{\beta}$ and $\Delta_{\alpha}$ decreases with the temperature and fall continuously to zero at $T_{N}$, which is a feature of a second-order phase transition. It should be noted that these values of $W$ are smaller than the value of the band-width at the point $\mathrm{C}$ of figure 1. However, for $W=1.00 \mathrm{eV}$, i.e., for $W$ located in the region where multiple solutions exist (as shown in Figure 1), although the gaps go to zero continuously at $\mathrm{T}_{\mathrm{N}}$, they exhibit a discontinuity at $k_{B} T \approx 0.0028 \mathrm{eV}$. For $W=1.025 \mathrm{eV}$, a similar behavior is observed, however, in this case, the discontinuity occurs at $k_{B} T \approx 0.0007 \mathrm{eV}$. This behavior is a manifestation of a first-order transition between the two competing SDW1 and SDW2. This finding is in accord with the results presented in
Figure 1. For $W=1.10$ and $1.20 \mathrm{eV}$, the gaps vanish continuously, as is also the case for $W=0.90 \mathrm{eV}$ and $W=$ $0.95 \mathrm{eV}$. For $W=1.650 \mathrm{eV}$ the gaps decrease with the temperature and fall discontinuously to zero at $T_{N}$. The inset of Figure 2(a) shows, in detail, the discontinuity of $\Delta_{\beta}$ at $k_{B} T \approx 0.0008 \mathrm{eV}$. A similar discontinuity of $\Delta_{\alpha}$ can be seen in Figure 2(b).

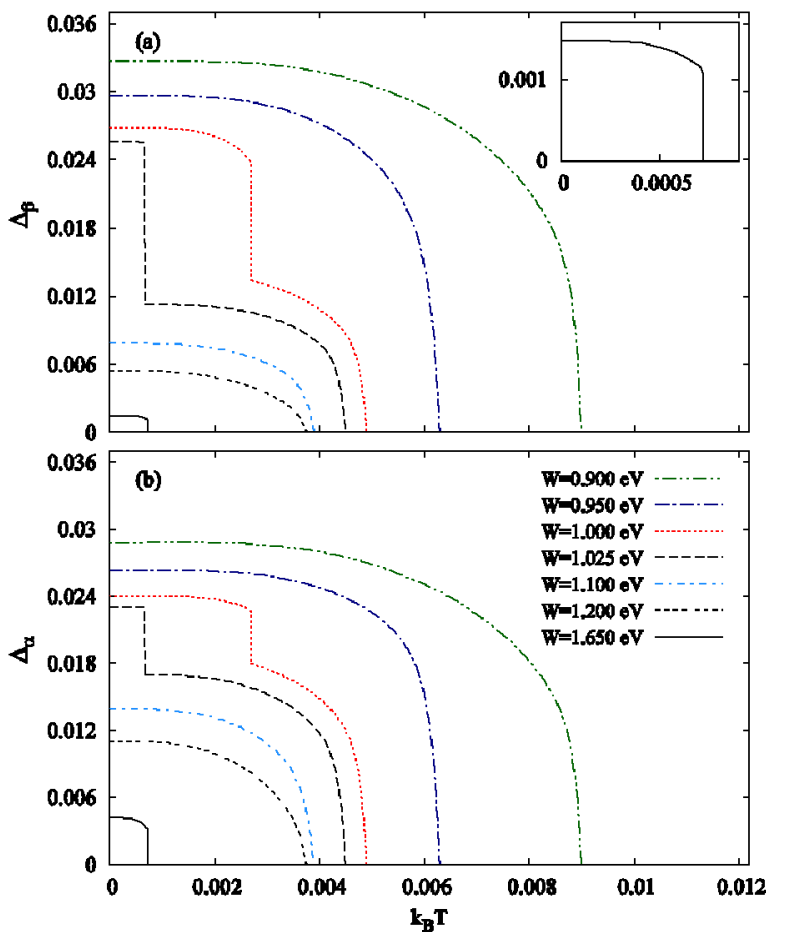

Figure 2. The upper panel (a) shows $\Delta_{\beta}$ as a function of temperature T, for various band-widths $W$. The lower panel (b) shows that $\Delta_{\alpha}$ exhibits similar behavior.

\section{Conclusions}

We have investigated the magnetic phase diagram of the UALM where the $5 \mathrm{f}$ bands are almost half-filled as a function of the $5 \mathrm{f}$ band-widths. The UALM supports Néel phases in which the ordering in one $5 \mathrm{f}$ band induces ordering in the second $5 \mathrm{f}$ band. As the $5 \mathrm{f}$ band-width increases, we find that the two gaps $\Delta_{\alpha}, \Delta_{\beta}$ and the induced sub-lattice magnetizations vary continuously but exhibit a distinct jump indicating a first-order transition between the two competing Néel phases. As the band-width increases, the tendency for magnetic order decreases, as is evidenced by a decrease in the Néel temperature, $T_{N}$. Initially, the paramagnetic state becomes unstable to the Néel state via a second-order instability at $T_{N}$. The decrease in $T_{N}$ with band-width suggests that the material will have a quantum critical point. However, before the system reaches the quantum critical point, the phase transition changes from second-order to first-order. The change of order of the transition is understood in terms of a slight mismatch between the position of the Fermi-energy and 
the energy at which the nesting would be optimal. The change in the order occurs when the energy lowering produced by the reconstruction of the Fermi-surface exceeds the entropy term in the Helmholtz Free-Energy. Since the mean-field approximation is not expected to be applicable in the critical region, defined by the Ginzburg criterion, the effects of commensurate and incommensurate fluctuations requires further investigation.

\section{Acknowledgements}

The authors would like to thank Professors J. Sereni and F. Honda for enlightening conversations. The work performed at Temple was supported by the US Department of Energy, Office of Basic Energy Sciences, Materials Science through award DE-FG02-01ER45872. This work was partially supported by the Brazilian agencies CNPq, CAPES and FAPERGS.

\section{References}

[1] $\mathrm{Ph}$. Nozières and A. Blandin, Kondo Effect in Real Metals, J. Physique, 41 (1980), pp. 193-211.

[2] J. Schoenes, B. Frick and O. Vogt, Transport properties of uranium monochalcogenide and monopnictide single crystals, Phys. Rev. B 30 (1984), pp. 6578-6585.

[3] J. Schoenes, O. Vogt, J. Lohle, F. Hulliger and K. Mattenberger, Variation of f-electron localization in diluted US and UTe, Phys. Rev. B 53 (1996), pp. 14987-14995.

[4] B. Coqblin, Magnetism and Kondo effect in cerium and uranium compounds, Acta Phys. Polonica A 113 (2008), pp. 391-394.

[5] N.B. Perkins, M.D. Nunez-Regueiro, J.R. Iglesias and B. Coqblin, Under-screened Kondo lattice model applied to heavy fermion uranium compounds, Phys. Rev. B 76 (2007), p.125101.

[6] C. Thomas, A.S. da Rosa Simões, J.R. Iglesias, C. Lacroix, N.B. Perkins and B. Coqblin, Application of the $\mathrm{S}=1$ under-screened Anderson lattice model to Kondo uranium and neptunium compounds, Phys. Rev. B 83 (2011), p.014415.

[7] P. Wisniewski, A. Gukasov and Z. Henkie, Spin and orbital moments in $\mathrm{U}_{3} \mathrm{X}_{4}$-type pnictides $(\mathrm{X}=\mathrm{P}$, As, Sb, Bi): Polarized neutron-diffraction study, Phys. Rev. B 60 (1999), pp. 6242-6245.

[8] T. Durakiewicz, J.J. Joyce, G.H. Lander, C.G. Olson, M.T. Butterfield, E. Guziewicz, A.J. Arko, L. Morales, J. Rebizant, K. Mattenberger and O. Vogt, Electronic structure of actinide antimonides and tellurides from photoelectron spectroscopy, Phys Rev. B 70 (2004), p.205103.

[9] F. Honda, Private Communication.

[10]P.S. Riseborough, B. Coqblin and S.G. Magalhães, Phase transition arising from the under-screened Anderson lattice model: a candidate concept for explaining hidden order in $\mathrm{URu}_{2} \mathrm{Si}_{2}$, Phys. Rev. $B$ 85 (2012), p.165116.

[11]E.J. Calegari, S.G. Magalhães and P.S. Riseborough, Unusual magnetic field-dependence of a possible hidden order phase, npj Quant. Mat. 2 (2017), 48. 